\title{
Magnifying chromoendoscopic and endocytoscopic findings of juvenile polyps in the colon and rectum
}

\author{
KENICHI TAKEDA ${ }^{1}$, SHIN-EI KUDO ${ }^{1}$, YUICHI MORI ${ }^{1}$, MASASHI MISAWA ${ }^{1}$, \\ TOYOKI KUDO ${ }^{1}$, KUNIHIKO WAKAMURA ${ }^{1}$, TAKEMASA HAYASHI ${ }^{1}$, \\ HIDEYUKI MIYACHI ${ }^{1}$, FUMIO ISHIDA ${ }^{1}$ and HARUHIRO INOUE ${ }^{2}$ \\ ${ }^{1}$ Digestive Disease Center, Showa University Northern Yokohama Hospital, Yokohama, Kanagawa 224-8503; \\ ${ }^{2}$ Digestive Disease Center, Showa University Koto-Toyosu Hospital, Tokyo 135-8577, Japan
}

Received October 29, 2014; Accepted August 27, 2015

DOI: $10.3892 / \mathrm{ol} .2015 .3910$

\begin{abstract}
A precise endoscopic diagnosis is necessary for endoscopic therapy for neoplastic and non-neoplastic lesions, including juvenile polyps (JPs). Therefore, the present study aimed to clarify the characteristic endoscopic findings of JPs. A total of 154 JPs were evaluated by magnifying chromoendoscopy, 20 of which were also assessed by endocytoscopy using an ultra-high magnification endoscope. Endoscopic images were evaluated in terms of gross appearance, color, pit pattern, surface inflammatory changes and vascularity of polyps. Endocytoscopic images were evaluated with regard to the morphology of glandular cavities, nuclei of glandular cells and interstitial features. Reddish surfaces (98.1\%), surface erosion (92.2\%), open pits $(90.3 \%)$ and low pit density (90.3\%) were observed in the majority of JPs by chromoendoscopy. In addition, dilated ductal openings surrounded by normal glandular cells $(100 \%)$, greater distances between gland basal layers $(100 \%)$ and interstitial infiltration by inflammatory cells $(100 \%)$ were observed in all JPs examined by endocytoscopy. These findings indicate that there is a tetralogy of magnifying chromoendoscopic findings characteristic of JPs: Reddish surfaces, surface erosion, open pits and low pit density. There is also a triad of endocytoscopic findings characteristic of JPs, namely dilated ductal openings surrounded by normal glandular cells, greater distances between gland basal layers, and interstitial infiltration by inflammatory cells. The aforementioned magnifying chromoendoscopic and endocytoscopic characteristics of JPs may be useful factors for diagnosing JPs.
\end{abstract}

Correspondence to: Dr Shin-Ei Kudo, Digestive Disease Center, Showa University Northern Yokohama Hospital, 35-1 Chigasaki-chuo, Tsuzuki, Yokohama, Kanagawa 224-8503, Japan

E-mail: kudos@med-showa-u.ac.jp

Key words: endocytoscopy, magnifying chromoendoscopy, ultra-high magnification, advanced endoscopy, juvenile polyp

\section{Introduction}

Juvenile polyps (JPs), which are considered to be a type of hamartomatous polyp (1), account for a small proportion of colorectal polyps. Solitary sporadic JPs are often observed in childhood, with a prevalence of $1-2 \%(2,3)$. When JPs meet the following criteria, juvenile polyposis syndrome (JPS), which is an autosomal dominant disorder characterized by the presence of multiple JPs in the gastrointestinal tract, is diagnosed: i) >3-5 JPs in the colorectum; ii) JPs throughout the intestinal tract; or iii) any number of JPs in combination with a positive family history of JPS (4). JPs are typically considered to be hamartomatous lesions with little malignant potential (5). JPS is reported as a risk factor for colorectal cancer and it has been reported that patients with JPS have a significantly elevated relative risk (34.0) of developing colorectal cancer compared with the general population (6).

Although certain studies have reported an association between their origin and genetic polymorphism, much remains uncertain, including why JPs originate from the colorectal mucosa, how they undergo malignant transformation and what the typical endoscopic and chromoendoscopic findings are $(7,8)$. In clinical practice, JPs may present as childhood intussusceptions or bloody stools, and they may also cause positive fecal occult blood tests in adulthood. JPs are usually detected incidentally as a result of colonoscopy for gastrointestinal tract complaints. Following the identification of JPs on endoscopic examination, lesions are resected endoscopically, and a definitive diagnosis is usually confirmed by pathological examination $(9,10)$.

Precise endoscopic diagnosis is important, however, there are few reports describing the endoscopic features of JPs (2). It is often difficult to differentiate JPs from adenomas, inflammatory myoglandular polyps, other hamartomatous polyps (such as solitary Peutz-Jeghers polyps), inflammatory polyps and hyperplastic polyps with inflammatory changes $(10,11)$.

Therefore, the present study retrospectively analyzed the endoscopic findings of JPs, particularly regarding the magnifying chromoendoscopic findings. In addition, the current study made the first attempt to evaluate JPs by endocytoscopy (EC). The EC system allows observation of living gastrointestinal cells, nuclei and vascularity in vivo under ultra-high 
Table I. Relevant patient characteristics and the gross appearance and endoscopic therapy of each juvenile polyp ${ }^{\mathrm{a}}$ (patients, $\mathrm{n}=128$; polyps, $\mathrm{n}=154)$.

\begin{tabular}{|c|c|c|c|}
\hline Characteristic & $\mathrm{MC}^{\mathrm{a}}$ & $\mathrm{MC}+\mathrm{EC}^{\mathrm{a}}$ & All patients \\
\hline \multicolumn{4}{|l|}{ Age, years } \\
\hline Mean & 47.2 & 46.7 & 47.1 \\
\hline Median & 47.0 & 47.5 & 47.0 \\
\hline Range & $2-88$ & $27-77$ & $2-88$ \\
\hline \multicolumn{4}{|l|}{ Gender, $\mathrm{n}$} \\
\hline Male & 91 & 5 & $96(75.0 \%)$ \\
\hline Female & 17 & 15 & $32(25.0 \%)$ \\
\hline \multicolumn{4}{|l|}{ Gross appearance, $\mathrm{n}$} \\
\hline Pedunculated & 53 & 9 & $62(40.3 \%)$ \\
\hline Semi-pedunculated & 49 & 8 & $57(37.0 \%)$ \\
\hline Non-pedunculated & 32 & 3 & $35(22.7 \%)$ \\
\hline \multicolumn{4}{|l|}{ Therapy, $\mathrm{n}$} \\
\hline Hot biopsy & 6 & 0 & $6(3.9 \%)$ \\
\hline Polypectomy & 90 & 12 & $102(66.2 \%)$ \\
\hline Endoscopic mucosal resection & 36 & 7 & $43(27.9 \%)$ \\
\hline Endoscopic piecemeal mucosal resection & 0 & 1 & $1(0.7 \%)$ \\
\hline Endoscopic submucosal dissection & 2 & 0 & $2(1.3 \%)$ \\
\hline
\end{tabular}

${ }^{\mathrm{a}}$ Lesions were resected endoscopically after being assessed by MC alone or by MC+EC. MC, magnifying chromoendoscopy; EC, endocytoscopy.

magnification, enabling real-time endoscopic assessment of histological characteristics. The present study was approved by the Ethics Committee of Showa University Northern Yokohama Hospital (Yokohama, Japan; approval no. 1509-02) and the requirement for consent was waived due to the retrospective nature of the study.

\section{Patients and methods}

Patients. A total of 154 JPs from 128 patients treated endoscopically at Showa University Northern Yokohama Hospital between April 2001 and April 2014 were assessed in the present study. Cases of JP diagnosed by colonoscopy were extracted from the hospital database. Of those cases, the diagnosis of JP was confirmed by histopathological diagnosis. In addition, endoscopic features of all lesions were observed by retrospectively assessing magnifying chromoendoscopy images captured prior to endoscopic resection, and all were ultimately diagnosed pathologically as JPs. Lesions that were pathologically diagnosed as hyperplastic polyp, inflammatory polyp and adenoma were excluded. In addition, 20 of these lesions from 16 patients were observed by EC following magnifying chromoendoscopy. Characteristic information of patients was obtained from patient history and interview data (Table I).

Assessment. Magnified chromoendoscopic images were retrospectively assessed to clarify the characteristic findings of JPs. To identify the characteristic findings, the evaluation focused on gross appearance, color, pit pattern and surface inflammatory changes. Furthermore, the morphology of glandular

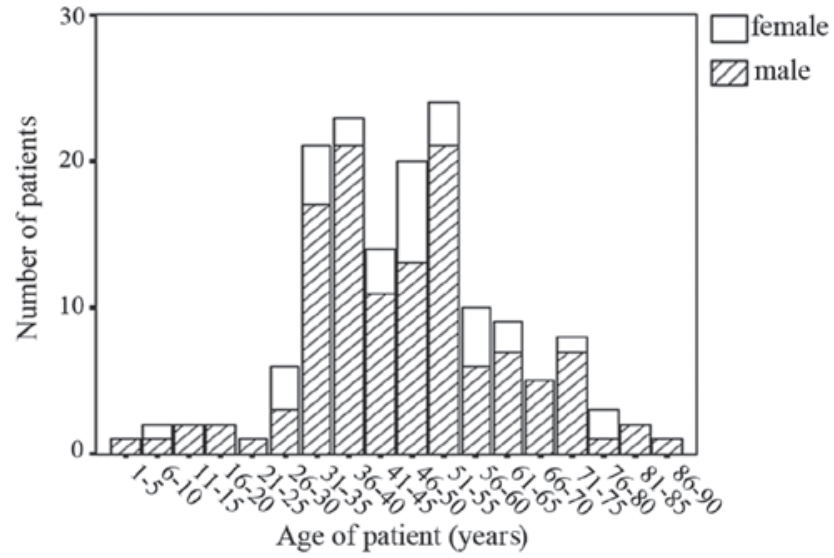

Figure 1. Age distribution of patients with juvenile polyps.

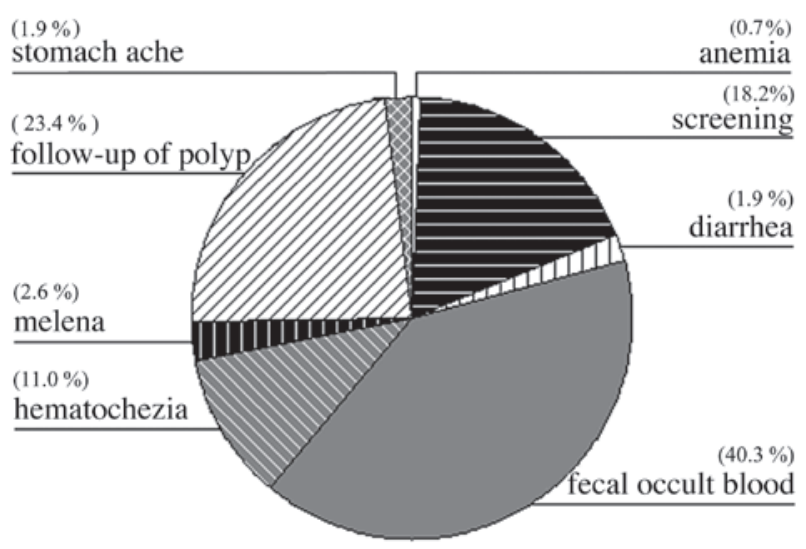

Figure 2. Presenting symptoms that prompted colonoscopic examination. 
Table II. Endoscopic findings of juvenile polyps ( $\mathrm{n}=154$ from 128 patients).

\begin{tabular}{|c|c|c|c|}
\hline Finding & $\mathrm{MC}^{\mathrm{a}}, \mathrm{n}$ & $\mathrm{MC}+\mathrm{EC}^{\mathrm{a}}, \mathrm{n}$ & All patients, n (\% \\
\hline \multicolumn{4}{|l|}{ Color } \\
\hline Reddish & 131 & 20 & $151(98.1)$ \\
\hline Non-reddish & 3 & 0 & $3(1.9)$ \\
\hline \multicolumn{4}{|l|}{ Pit pattern } \\
\hline Type I & 0 & 1 & $1(0.7)$ \\
\hline Type II & 122 & 18 & $140(90.9)$ \\
\hline Type III-L & 11 & 0 & $11(7.1)$ \\
\hline Type IVb & 1 & 1 & $2(1.3)$ \\
\hline \multicolumn{4}{|l|}{ Open pit } \\
\hline Present & 119 & 20 & $139(90.3)$ \\
\hline Absent & 15 & 0 & $15(9.7)$ \\
\hline \multicolumn{4}{|l|}{ Mucus cap } \\
\hline Present & 37 & 8 & $45(29.2)$ \\
\hline Absent & 97 & 12 & $109(70.8)$ \\
\hline \multicolumn{4}{|c|}{ Lobular appearance } \\
\hline Present & 17 & 5 & $22(14.3)$ \\
\hline Absent & 117 & 15 & $132(85.7)$ \\
\hline \multicolumn{4}{|c|}{ Decreased pit density } \\
\hline Present & 119 & 20 & $139(90.3)$ \\
\hline Absent & 15 & 0 & $15(9.7)$ \\
\hline \multicolumn{4}{|l|}{ Surface erosion } \\
\hline Present & 122 & 20 & $142(92.2)$ \\
\hline Absent & 12 & 0 & $12(7.8)$ \\
\hline \multicolumn{4}{|c|}{ Increase of vascularity } \\
\hline Present & 128 & 20 & $148(96.1)$ \\
\hline Absent & 6 & 0 & $6(3.9)$ \\
\hline
\end{tabular}

${ }^{a}$ The lesions were resected endoscopically after being assessed by MC alone or by MC+EC. MC, magnifying chromoendoscopy; EC, endocytoscopy.

cavities, nuclei of glandular cells and interstitial features were also evaluated in EC images, and the EC findings were compared with findings on conventional pathological examination.

Each magnified chromoendoscopic image was obtained using a CF-Q240Z or CF-H260AZI (Olympus Corporation, Tokyo, Japan) instrument. The ultra-high magnification images were obtained with an integrated type endocytoscope (CF-Y0020-I, prototype; Olympus Corporation). The endocytoscope has a single lens on its tip with a hand lever, enabling the magnifying power of conventional endoscopic images to be increased to the ultra-high magnification power of $\mathrm{x} 380$, which covers a $700 \times 600 \mu \mathrm{m}$ area of tissue.

To obtain the magnified chromoendoscopic images, indigo carmine (Nagase Medicals Co., Ltd., Hyogo, Japan) was sprayed directly onto lesions through an endoscope instrument channel or using a non-traumatic catheter (12) (Olympus Corporation). To obtain the EC images, the non-traumatic catheter was subseqeuntly used to spray $0.05 \%$ crystal violet (Koso Chemical Co., Ltd., Gyoda, Japan) and 1.0\% methylene blue dyes (Wako Pure Chemical Industries Ltd., Osaka, Japan)
Table III. Endocytoscopic findings of juvenile polyps $(n=20)$.

\begin{tabular}{lc}
\hline Finding & $\mathrm{n}(\%)$ \\
\hline Endocytoscopy classification & $1(5.0)$ \\
$1 \mathrm{a}$ & $19(95.0)$ \\
$1 \mathrm{~b}$ & \\
Atypical cells & $0(0.0)$ \\
Present & $20(100.0)$ \\
Absent & \\
Dilated opening duct & $20(100.0)$ \\
Present & $0(0.0)$ \\
Absent & \\
Elongated distance between gland basal layers & $20(100.0)$ \\
Present & $0(0.0)$ \\
Absent & \\
Interstitial infiltration of inflammatory cells & $20(100.0)$ \\
Present & $0(0.0)$ \\
Absent &
\end{tabular}


A

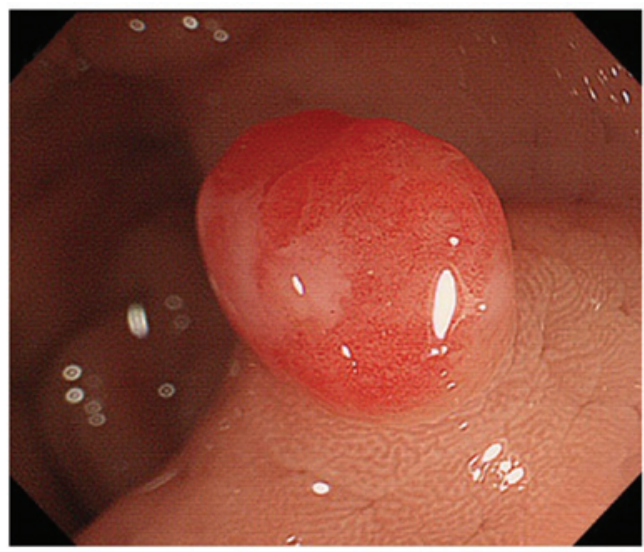

C

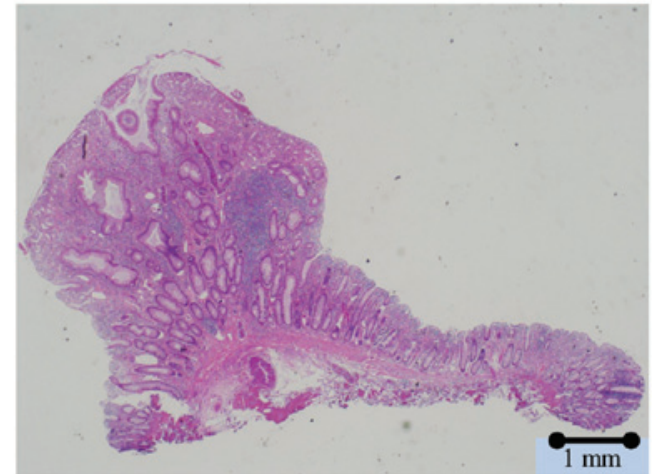

B

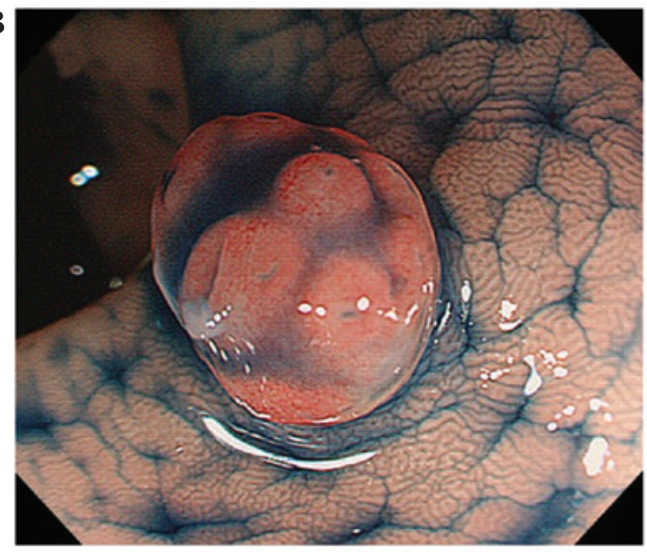

D

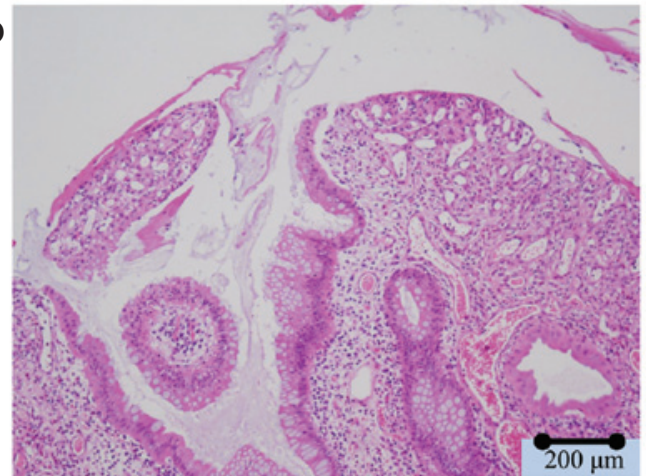

Figure 3. Endoscopic images (A and B) and microphotographs of hematoxylin and eosin-stained images (C and D) of juvenile polyp (diameter, 5 mm). (A) Endoscopic images of juvenile polyps in white light and (B) after spraying with indigo carmine. Caps of white mucus adhering to the polyp are apparent, as are surface erosions and low pit density in the epithelium between glandular cavities. (C and D) Microphotographs of hematoxylin and eosin-stained images of juvenile polyp show the characteristic findings, namely, erosive changes in the surface epithelium, cystic dilated glandular ducts, inflammatory cell infiltration and proliferation of blood vessels. (D) Surface of juvenile polyp is erosive and epithelium layer of polyp are fallen away. Cystic dilated gland is filled with mucus.
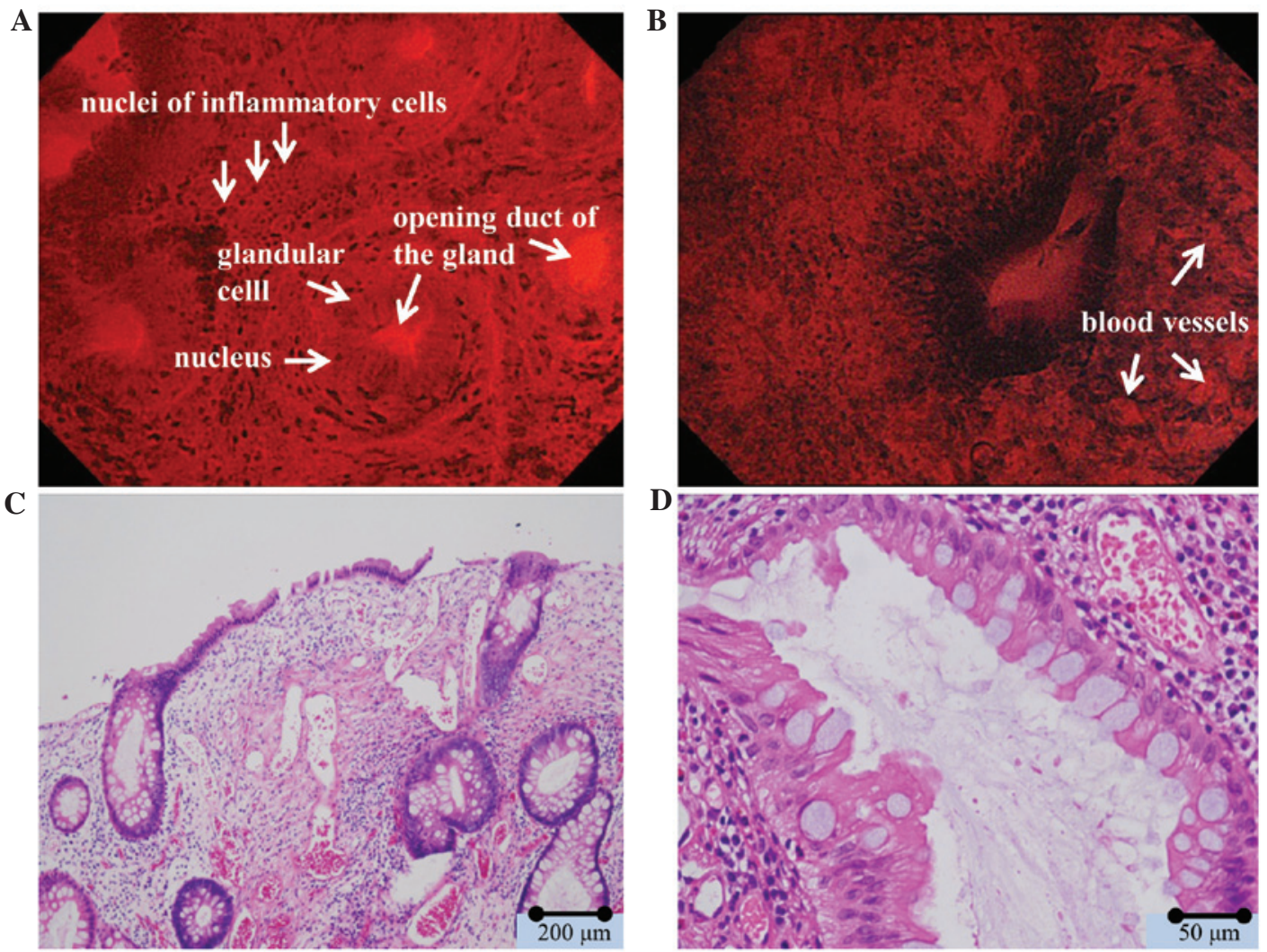

Figure 4. Endocytoscopy images (A and B) and microphotographs of hematoxylin and eosin-stained images (C and D) of juvenile polyp. (A and B) Inflammatory cell infiltration, proliferation of blood vessels, and cystic dilated glandular ducts are visible. (C) There is a low density of pits, which means the distances between gland basal layers are greater than in normal colonic mucosa. (B and D) EC image well reflects the characteristic features of juvenile polyp. 
softly onto lesion to stain the cell cytoplasm and cell nuclei, respectively.

To evaluate the lesions, the pit pattern classification for magnifying chromoendoscopy (13) and the EC classification for EC (14) were used. The pit pattern classification relies on the features of the glandular duct lumen: Type I, round pits, regular in size; Type II, larger in size than normal, star-shaped or onion-like pits; Type IIIs, lesions with compactly arranged pits, smaller in size than normal ones; TypeIII-L, elongated pit; Type IV, branched pit; Type V, irregular pit (13). We further divided Type IV pit patterns into Type IVb (branch-like) and Type IVv (villous-like) lesions. The EC classification, relies on the features of glandular duct lumens and the shape of nuclei in the superficial layers of tumors: ECla, rounded lumens and fusiform nuclei; EC1b, narrow, serrated lumens and small rounded nuclei; EC2, slit-like, smooth lumens and uniform fusiform or rounded nuclei; EC3a, irregular and rough lumens and a large number of rounded nuclei; EC3b, unclear gland formation and agglomeration of distorted nuclei (14).

\section{Results}

Patient characteristics. A total of 128 patients, comprising 96 males and 32 females with a mean age of 47.1 years, a median age of 47 years, and an age range of 2-88 years, were included in the study (Fig. 1; Table I). The most common symptomatic presentations were associated with bleeding from the gastrointestinal tract [total, 53.9\%; melena (2.6\%); hematochezia (11.0\%); detection of fecal occult blood (40.3\%)] (Fig. 2).

Lesion characteristics. Of the 154 polyps assessed, the median size of the lesions was $11.6 \mathrm{~mm}$. With regard to location, 3 lesions were detected in the cecum, 25 in the ascending colon, 19 in the transverse colon, 8 in the descending colon, 59 in the sigmoid colon and 40 in the rectum. In terms of gross appearance, 62 of the lesions were pedunculated polyps, 57 semi-pedunculated polyps and 35 non-pedunculated polyps. Sporadic JPs accounted for 126 of the cases. There were only 2 cases of JPS with multiple polypoid polyps in the colorectum. The polyps were treated by hot biopsy in 6 cases, polypectomy in 102 cases, endoscopic mucosal resection in 43 cases, endoscopic piecemeal mucosal resection in 1 case and endoscopic submucosal dissection in 2 cases (Table I). No adenomatous or malignant changes were detected in any of the JPs.

Endoscopic findings. In white light, 151 polyps were observed to have reddish surfaces. Caps of white mucus adhering to the surfaces of the polyps were observed in 45 lesions (Fig. 3) and reddish areas, reflecting an increase in vascularity surrounding the pits were present in 148 lesions. Type II pit patterns were observed in 140 lesions, type III L pit patterns in 11 lesions, and a type $\mathrm{IVb}$ pit pattern in 2 lesions. There were frequent, relatively small, round pits in the majority of the polyps with the type II pit pattern. Open pits were observed in 139 lesions. There were 22 lobulated polyps. Low pit density was observed in 139 lesions. Erosion was observed in epithelial cells between glandular cavities in 142 lesions (Fig. 3; Table II).

EC findings. Of the 20 polyps observed by EC, 1 polyp was classified as ECla and 19 polyps as EClb according to the EC classification criteria. No atypical changes were observed in the nuclei of the glandular cells of any polyps. A number of the glandular cavities were dilated by abundant mucus (Fig. 4). In addition, numerous compact nuclei, which were considered to be nuclei of inflammatory cells, were observed in the intercellular substance between glandular cells (Fig. 4; Table III).

\section{Discussion}

The pathological characteristics of JPs are erosive changes in the surface epithelium, inflammatory cell infiltration, proliferation of blood vessels and cystic dilated glandular ducts (4). These characteristics are readily identifiable by optical microscopy, in which desquamated epithelium consequent to inflammatory changes in the surfaces of the polyps, serrated and cystic dilated glandular ducts, and infiltration by inflammatory cells, such as lymphocytes, are clearly visible (4). However, we hypothesize that it is often difficult to visualize these pathological characteristics endoscopically.

According to the current findings, the characteristic endoscopic features of JPs are reddish surfaces, surface erosion, caps of white mucus on the surfaces, open pits surrounded by inflammatory changes, and low pit density. When the endoscopic and pathological findings were compared, they were found to correspond. The inflammatory changes lead to epithelial erosion and account for the colonoscopic findings of reddish surfaces and caps of white mucus adhering to the polyps (4). The open pits visible by colonoscopy correspond with the cystic dilated glandular ducts filled with mucus observed pathologically. The low pit density is caused by the increased interstitial volume and cystic dilated glandular ducts stretching the polyp surfaces (11). Thus, the colonoscopic findings accurately reflect the pathological findings. It may therefore be concluded that the following tetralogy of endoscopic findings, which is found in most JPs, is characteristic of these polyps: i) reddish surfaces; ii) surface erosion; iii) open pits; and iv) low pit density.

The observations provided by EC assessment of the JPs were also consistent with the pathological findings. On EC, the areas that had appeared inflamed and reddish on magnifying chromoendoscopy were observed to contain increased blood vessels, particularly surrounding the pits. These findings suggest that the following triad of EC findings is characteristic of JPs: i) dilated ductal openings surrounded by normal glandular cells; ii) greater distances between gland basal layers; and iii) interstitial infiltration by inflammatory cells.

Patients with JPS are known to be at increased risk of colorectal cancer $(6,15)$. Adenomatous or malignant changes have been observed in subjects with JPS and also in those with sporadic JPs $(16,17)$. In the present study, no malignant changes were detected; nonetheless, EC is able to detect malignant changes as it enables the assessment of nuclei in real time. A number of studies have reported the usefulness of EC in the diagnosis of epithelial neoplastic lesions $(14,18,19)$. The current study supports previously reported endoscopic findings (6). However, to the best of our knowledge, no previous studies have described the use of EC with regard to JPs. Upon comparison of current EC results with previous pathological findings, EC data was found to accurately reflect the pathological findings $(4,11)$. Considering that the current findings indicate that EC images 
accurately reflect the pathological findings of JPs, EC may be of value, not only for diagnosing neoplastic lesions, but also for diagnosing non-neoplastic lesions including JPs. JP has similar endoscopic features to other polyps, such as inflammatory and hyperplastic polyps; therefore, clarifying the features of magnifying endoscopic and EC findings will aid in differentiating between these disease entities (11).

Although JPs are relatively rare, they are occasionally discovered in daily medical practice, particularly by colonoscopic examination (20). It is important to make a precise diagnosis to enable optimal treatment (5). The risk of bleeding after colonoscopic polypectomy was reported as $0.3-6.1 \%$ (21). Precise endoscopic diagnosis will make it possible to avoid endoscopic biopsy or resection for confirming pathological histology, and, thus, enable the risk of bleeding caused to be reduced, particularly in patients that have been administered anticoagulant treatments. Furthermore, although the malignant potential of JPs not particularly high, their diagnosis of JPs on the basis of endoscopic features is important, as it allows clinicians to consider whether immediate treatment is required (5).

Precise endoscopic diagnosis prevents unnecessary biopsy or resection, and reduces the risk of bleeding caused by treatment. Therefore, the present findings are of great significance in the endoscopic diagnosis and management of JPs.

\section{Acknowledgements}

The authors wish to thank Edanz Group Ltd. (Fukuoka, Japan) for assistance with correcting English in this paper.

\section{References}

1. Morson BC: Precancerous and early malignant lesions of the large intestine. Br J Surg 55: 725-731, 1968.

2. Brosens LA, Langeveld D, van Hattem WA, Giardiello FM and Offerhaus GJ: Juvenile polyposis syndrome. World J Gastroenterol 17: 4839-4844, 2011.

3. Cichy W, Klincewicz B and Plawski A: Juvenile polyposis syndrome. Arch Med Sci 10: 570-577, 2014.

4. van Hattem WA, Langeveld D, de Leng WW, Morsink FH, van Diest PJ, Iacobuzio-Donahue CA, Giardiello FM, Offerhaus GJ and Brosens LA: Histologic variations in juvenile polyp phenotype correlate with genetic defect underlying juvenile polyposis. Am J Surg Pathol 35: 530-536, 2011.

5. Giardiello FM, Hamilton SR, Kern SE, et al: Colorectal neoplasia in juvenile polyposis or juvenile polyps. Arch Dis Child 66: 971-975, 1991.
6. Brosens LA, van Hattem A, Hylind LM, Iacobuzio-Donahue C, Romans KE, Axilbund J, Cruz-Correa M, Tersmette AC, Offerhaus GJ and Giardiello FM: Risk of colorectal cancer in juvenile polyposis. Gut 56: 965-967, 2007.

7. Barros R, Mendes N, Howe JR, Reis CA, de Bolos C, Carneiro F, David L and Almeida R: Juvenile polyps have gastric differentiation with MUC5AC expression and downregulation of CDX2 and SMAD4. Histochem Cell Biol 131: 765-772, 2009.

8. Marsh Durban V, Jansen M, Davies EJ, Morsink FH, Offerhaus GJ and Clarke AR: Epithelial-specific loss of PTEN results in colorectal juvenile polyp formation and invasive cancer. Am J Pathol 184: 86-91, 2014.

9. Stojcev Z, Borun P, Hermann J, Krokowicz P, Cichy W, Kubaszewski L, Banasiewicz T and Plawski A: Hamrtomatous polyposis syndromes. Hered Cancer Clin Pract 11: 4, 2013.

10. Shussman N and Wexner SD: Colorectal polyps and polyposis syndromes. Gastroenterol Rep (Oxf) 2: 1-15, 2014.

11. Ricci MT, Salemme M, Villanacci V and Varesco L: The genetics of inherited predispositions to colorectal polyps: A quick guide for clinicians. Colorectal Dis 17 (Suppl 1): S3-S9, 2015.

12. Fujii T, Hasegawa RT, Saitoh Y, et al: Chromoscopy during colonoscopy. Endoscopy 33: 1036-1041, 2001.

13. Kudo S, Rubio CA, Teixeira CR, Kashida H and Kogure E: Pit pattern in colorectal neoplasia: Endoscopic magnifying view. Endoscopy 33: 367-373, 2001.

14. Kudo S, Wakamura K, Ikehara N, Mori Y, Inoue H and Hamatani S: Diagnosis of colorectal lesions with a novel endocytoscopic classification-a pilot study. Endoscopy 43: 869-875, 2011.

15. Howe JR, Mitros FA and Summers RW: The risk of gastrointestinal carcinoma in familial juvenile polyposis. Ann Surg Oncol 5: 751-756, 1998.

16. Friedman CJ and Fechner RE: A solitaly juvenile polyp with hyperplastic and adenomatous glands. Dig Dis Sci 27: 946-948, 1982.

17. Kang SH, Chung WS, Hyun CL, Moon HS, Lee ES, Kim SH, Sung JK, Lee BS and Jeong HY: A rare case of a signet ring cell carcinoma of the colon mimicking a juvenile polyp. Gut Liver 6: 129-131, 2012.

18. Inoue H, Kudo SE and Shiokawa A: Technology insight: Laser-scanning confocal microscopy and endocytoscopy for cellular observation of the gastrointestinal tract. Nat clin Pract Gastroenterol Hepatol 2: 31-37, 2005.

19. Mori Y, Kudo S, Ikehara N, Wakamura K, Wada Y, Kutsukawa M, Misawa M,Kudo T, Kobayashi Y,Miyachi H, et al: Comprehensive diagnostic ability of endocytoscopy compared with biopsy for colorectal neoplasms: A prospective randomized noninferiority trial. Endoscopy 45: 98-105, 2013.

20. Howe JR, Sayed MG, Ahmed AF, Ringold J, Larsen-Haidle J, Merg A, Mitros FA, Vaccaro CA, Petersen GM, Giardiello FM, et al: The prevalence of MADH4 and BMPR1A mutations in juvenile polyposis and absence of BMPR2, BMPR1B, and ACVR1 mutations. J Med Genet 41: 484-491, 2004.

21. Rosen L, Bub DS, Reed JF 3rd and Nastasee SA: Hemorrhage following colonoscopic polypectomy. Dis Colon Rectum 36: 1126-1131, 1993. 